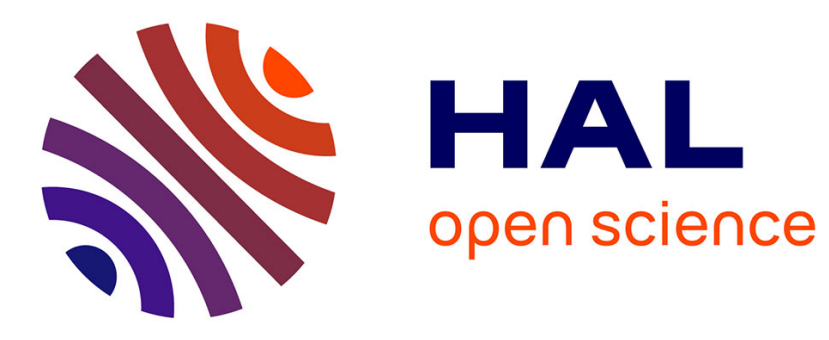

\title{
A methodology to identify efficient collaborative practices of decision-making in industrial projects, based on enterprise process modeling
}

Saïna Hassanzadeh, Didier Gourc, François Marmier, Sophie Bougaret

\section{- To cite this version:}

Saïna Hassanzadeh, Didier Gourc, François Marmier, Sophie Bougaret. A methodology to identify efficient collaborative practices of decision-making in industrial projects, based on enterprise process modeling. INCOM 2012 - 14th IFAC Symposium on Information Control Problems in Manufacturing, May 2012, Bucharest, Romania, Romania. pp.1339-1346. hal-00746442

\section{HAL Id: hal-00746442 \\ https://hal.science/hal-00746442}

Submitted on 29 Oct 2012

HAL is a multi-disciplinary open access archive for the deposit and dissemination of scientific research documents, whether they are published or not. The documents may come from teaching and research institutions in France or abroad, or from public or private research centers.
L'archive ouverte pluridisciplinaire HAL, est destinée au dépôt et à la diffusion de documents scientifiques de niveau recherche, publiés ou non, émanant des établissements d'enseignement et de recherche français ou étrangers, des laboratoires publics ou privés. 


\title{
A methodology to identify efficient collaborative practices of decision-making in industrial projects, based on enterprise process modeling ${ }^{\star}$
}

\author{
S. Hassanzadeh * D. Gourc* F. Marmier* S. Bougaret ${ }^{* *}$ \\ * Université de Toulouse, Mines Albi, Centre Génie Industriel, Campus \\ Jarlard,81013 Albi,France (e-mail: saina.hassanzadeh@mines-albi.fr). \\ ** Pharmaceutical RED Management Consulting Company, Manageos, \\ Le moulin de Souleilla, 31460 Francarville, France (e-mail: \\ sophie.bougaret@wanadoo.fr)
}

\begin{abstract}
Industrial projects are often difficult to accomplish, reaching all their goals on quality, time, cost, and respecting human well-being. Making decisions effectively and without delays would help achieve the goals and prevent waste of human and material resources. However, as recent literature shows very few organizations have undertaken systematic efforts to enhance decision-making processes. This paper investigates a methodology to identify and structure efficient practices that would improve collaborative decision-making in industrial projects. The proposed methodology uses CIMOSA cube as a reference architecture that provides multiple views to analyze the different aspects of decision-making process. A case study in drug development projects is presented to illustrate the interest of the proposed approach.
\end{abstract}

Keywords: enterprise engineering, enterprise reference models, collaborative practices, decision-making

\section{INTRODUCTION}

In complex industrial projects, make decisions without delays helps achieve the goals of the projects. Nevertheless, some research show that "relatively few businesses and organizations have given full and proper attention to one of their most important activities: decision-making... very few organizations have undertaken systematic efforts to improve a variety of decisions" (Davenport (2010)). Some astonishing examples of unsuccessful decision-making processes are given in literature, including 17 years of stops and starts in the project of construction of San-FranciscoOkland Bay Bridge, 20 years in a project of personal public transportation in Paris, called Aramis that was ever actually completed, and 30 years in the new international airport in Lisbon. Indecision culture at Yahoo, Airbus, and Boeing are other examples (Denis et al. (2011)).

Several questions arise from the mentioned examples. Why decisions are frequently delayed in some projects? Why made decisions are not respected and are unmade and remade over many years? What are the decisionmaking processes applied in these projects? What are the information on which these decisions are based? Who are the actors that contribute to these decisions? What are their tasks, resources, and responsibilities in decisionmaking? How do they collaborate? What are the causes and the remedies to indecision problem? In this paper, a methodology based on enterprise process modeling is

\footnotetext{
* This work was supported by The Foundation for an Industrial
} Safety Culture (Fondation pour une Culture de Sécurité Industrielle). proposed that attempts to answer these questions in a given industrial context. To reply these questions, there is a need for a reference architecture that gives the key elements of these questions a structure. The CIMOSA cube as a reference architecture is used, providing multiple views to analyze the different aspects of the decisionmaking processes in industrial projects.

The proposed methodology is applied to indecision problem in pharmaceutical industry. As the actors of the pharmaceutical industry complain, in pharmaceutical R\&D projects, either decisions are lengthy postponed, before reaching a compromise between decision-makers, or decisions are frequently invalidated, a posteriori. The application of the methodology to this problem helps identify the efficient practices of collaborative decision-making. The analysis of the efficient practices, recommended by the pharmaceutical actors, shows that the subjective and contextual influential factors play an important role in decision-making process. However, these factors are few taken into account in enterprises.

The structure of this paper is as follows. In section 2, a brief literature review on process modeling in enterprise is presented. In section 3 , the different steps of the proposed methodology is presented. In section 4, the methodology is applied to indecision problem in drug development projects. In section 4 , the conclusions, the limitations of the methodology, and also the opportunities for further research are summarized. 


\section{LITERATURE REVIEW}

Indecision in projects adversely affects achieving objectives on quality, time, and cost as planned. Enterprise engineering provides industrial actors with enterprise reference models that help analyse, restructure, and improve the performance of enterprises (Vernadat (1996)). In all cases, the purpose is to understand the functioning of an enterprise, either to help decision-making, or to improve the functioning (Hammer and Champy (1993)).

Methods and tools for enterprise modeling have appeared under different names since the early 80's. Among the first works, Integrated Computer Aided Manufacturing (ICAM) of the US Air Force program is cited that produced at that time the ICAM DEFinition (IDEF) modeling languages, based on Structured Analysis Design Technique (SADT) (Darras (2004); Ferchichi (2008)). In the early 90 's, sharing representative frameworks between different industrial actors, using enterprise modeling, has became a general trend (Darras (2004)). Several architectures and methods for process modeling are then proposed such as CIMOSA, Zachman, MERISE, and GRAI (Ferchichi (2008)).

Computer Integrated Manufacturing Open System Architecture (CIMOSA) (AMICE (1993)) is one of the enterprise reference architecture that helps analyze integrated production systems. The advantage of CIMOSA is its generation axis that proposes different points of view: 1) function view: activities and tasks, 2 ) information view: objects of the enterprise, 3) resources view: material and human resources, and 4) organization view: hierarchical relations and responsibilities. In complex industrial contexts, these multiple points of view allow: first, to filter some aspects of the enterprise, in order to temporarily concentrate on one aspect, and then to make relationships of different aspects. The different aspects of decision-making process such as tasks, information, actors and their roles and relationships can be structured according to the generation axis. In order to improve a process, it is necessary to review the sequence of the tasks and also the way in which the tasks are done. But the notion of practice as a way of doing a task is not included in the forth primary views. The ISO19439 norm states if it is necessary an additional modeling view can be added to the CIMOSA cube. In this paper, practice view is added in order to study the practices implemented by decision-makers. A collaborative practice refers to the way of doing a task by a set of actors who collaborate to carry out tasks, to reach a common goal.

In next section, based on the forth primary views of the CIMOSA cube and the additional view of practices, a methodology is proposed to identify the causes of delays in collaborative decision-making and the efficient practices to reduce these delays.

\section{PROPOSED APPROACH}

The industrial projects are defined to attain the enterprise's goals such as a product or a service. These projects are usually composed of different phases with intermediate goals that are indispensable to achieve the final goals. In this way, at the end of each phase, transition decision to the next phase depend on intermediate goals such as conception of a product, transformation of a raw material, definition a service, validation a plan. The transition decisions are usually made by a group of actors who collaborate to reach the project's goals. Attaining the project's goals depend on the efficiency of the collaboration of actors in decision-making process. If the decisions are frequently not delayed, this collaboration is not efficient.

In this paper, a methodology is proposed to identify the efficient practices for collaborative decision-making, based on process modeling. This methodology aims first to identify the causes of delays in decision-making and then to improve the efficiency of an enterprise wherein expected goals are frequently not achieved in the expected lapse of time by: 1) providing the enterprise reference models that can support the coherency inside of an enterprise, 2) proposing a set of structured efficient practices that facilitate and accelerate the process of collaborative decisionmaking.

In this section, first, the main steps of the proposed methodology are described. Second, a case study in drug development projects is illustrated.

\subsection{Main steps of the proposed approach}

The main steps of the proposed methodology are presented in a form of a process in figure A.1 in appendix. The explanations of each step are developed here.

Identification of a problem The proposed methodology can be triggered by identification of a chronic difficulty in decision-making by the actors of an industrial field. The main pathology is frequent delays in decision-making result from either postponement of decisions or invalidation of decisions. The symptoms characterize this pathology at two levels. At individual level, the symptoms of indecision may be: feelings of doubt about the accuracy and completeness of the available information and the pertinence of the eventually selected alternative (fear of mistakes), worry about the consequences of the decision (undesirable effects), and regret about what would be lost (missing opportunities) Germeijs and De Boeck (2002); Bacanli (2006). At collaborative level, the symptoms may be: lack of discussion or endless contradictory discussions in meetings, hold and re-hold meetings for the same decisions on the same information, lack of determination in execution of a decision.

In sum, if the gap between expected and real time to make decisions is unacceptable, decision-making process has to be revised, in order to improve the performance in future.

Characterization of the problem The characterization of indecision problem helps to examine its particularities in a given industrial context. The common point of indecision problems may be the result means delays in decisions. But each indecision problem has its own characteristics that repeat in each indecision situation. During the characterization of the problem, the key element is to detect the set of specificities that distinguish an indecision problem from similar problems in other industries. This characterization may be guided by verification of some factors related to the decisions: subject of decision, type of decision (individual 
or collaborative), type and phases of the projects, presence of uncertainty, time for decision-making, etc. The specificities of the industrial field may add other characteristics to the problem.

Knowledge acquisition on the industrial field "Things are better controlled if they are better understood" (Vernadat (1996)). Acquisition knowledge on the given industrial field and its specificities helps to understand the context of the projects. Internal reports, bibliographical and field studies, involving interviewing and observation are some potential sources of knowledge that first help to describe and model the decision-making process and then to improve it.

Modeling a generic process "Better processes won't guarantee better decisions, of course, but they can make them more likely" (Davenport (2009)). The purpose of this step is providing a generic process that helps coherency and transparency inside of the enterprise. Different points of view of the CIMOSA cube provide a framework of modeling: function view, information view, resources view, and organization view. These views are the axes of the first series of interviews that aim to describe/model the tasks that have to be performed, the information flow, the actors who contribute to the decisions, and their relationships. Second series of the interviews aim to identify the causes of delays in decision-making and the efficient practices for the "practices view" that allows to improve the way of doing the tasks. The axes of the second series of interviews should be identified.

Identification of the axes of interviews The characteristics of the problem and the generic process, provided in previous steps, help to determine the main axes of the interviews. First, the tasks or the loops whose objectives are not satisfactory or not attained in the expected time are identified. Second, the influential factors or the characteristics of the problem that may play a role on the difficulties of these tasks are detected and grouped in the axes of interviews.

Identification of the target actors In previous steps the tasks or the loops, whose objectives are not attained, are identified. These tasks are performed by different actors. The purpose of this step is to cross the visions of the different actors in order to understand the points of disagreement and misunderstanding between them. The actors are then grouped according to their: 1) professions, 2) psychological profiles, or 3) contributions in product life cycle. Each of these groups may help to understand the point of view of a type of actors in collaborative decisionmaking process.

Development of the interview grid Interviews' questions have to provide the opportunities for interviewees to explain the problems. The main axes of the interview are detailed in this step, developing: the impact of the problem's characteristics, the difficulties of each type of tasks, and the problems of each type of actors. In each axis, the first question is about the importance of the axis' theme. The first question should be general enough, in order to do not influence the interviewees by an idea. Doing so, the interviewees can begin to reply by the most importance aspects, according to their experience. The last question can be an open question that allows to interviewees to add an opinion that was not included in the questions or to give a summary of their point of view. Between these two questions, the other questions may be on the causes of the non acquisition of the objectives, the difficulties, the influential factors, the overestimated spent time and costs in the problematical task or loops, and finally the improvements and the possible barriers.

Validation of the grid The grid is reviewed by some of the industrial actors. They propose the improvements, adding questions, using the words that industrial actors are used to use, removing the ambiguities and adding some definitions or precision, if necessary.

Interviews The objective is to collect the unspoken information related to the difficulties of decisions and possible improvements. The interviewees should feel free to criticize the actual practices. One of the difficulties is to create an atmosphere of confidence for the industrial actors. Recording the interviewees can harm this confidence. It is also important to plan a sufficient timing for interviews and to make contact with them, in order to complete the interviews.

Identification and structuring the efficient practices Influential factors on the decision-making and causes of delays are identified in interviews. With each influential factor, efficient practices are associated that help controlling this factor. These factors are then classified into three main classes: 1) subjective factors: individual such as psychological properties and collaborative factors such as contradictory opinions, 2) objective factors: information, uncertainties, risks related to the project's goals, 3) contextual factors: internal factors inside of the enterprise such as the organizational and hierarchical factors and the external factors related to the changes in the external environment such as market dynamics, competitors' activities, stakeholders' expectations. These classes take root in a typology of influential factors on generation and processing of uncertainty, proposed by Hassanzadeh et al. (2012).

Evaluation of the solution Solution refers to both the generic process and the efficient practices. The effectiveness, coherency, acceptability, applicability of the process and the practices, and the barriers to apply them should be evaluated by the industrial engineering actors. The process and the efficient practices are strongly interconnected and any change in process implies review the practices and vice versa.

\subsection{Illustration of an example in drug development projects}

Identification of a problem Drug development projects are composed of different phases of tests and studies on safety, efficacy, and quality of presumed new medicines. At the end of each phase, based on the results of these tests, a steering committee has to decide whether to continue or to stop the project. According to pharmaceutical actors, these transition decisions, called Go / No Go decisions are lengthy delayed, or they are invalidated frequently, or some of them are never made and the projects are "left to rot", as a project manager affirm. 
Characterization of the problem The problem is that the collaborative decisions are usually postponed face of uncertainty. This problem is characterized by three key elements that repeat in each Go / No Go decision: a high degree of uncertainty, non-emergency of situations, and collaborative decisions. 1) a strong degree of uncertainty: in spite of all numerous tests and studies, the degree of uncertainty about the behavior of the new compounds in the human body is generally high. For example, the tests performed on animals in preclinical phase may be inconclusive to begin tests on human in clinical phase???. The benefits and risks of the molecule, means its helpful and harmful affects, cannot be known completely, 2) non-emergency situations: R\&D context, compared to emergency situations such as an accident, crisis, and disaster contexts is not emergency, since the consequences of the decisions are not immediate. In emergency situation, decision-makers rapidly make a decision based on the piece of information that is available. In non-emergency situation, decision-makers waiting for a more accurate information , 3) the collaborative aspect: Go/No Go decisions are made by a multidisciplinary steering committee composed of the pharmacists, financial, and marketing experts. Individual differences within a group play a crucial role in interactions between experts and may complicate the decision (or indecision) process and increase uncertainty.

Knowledge acquisition on the industrial field The purpose of pharmaceutical industry is to research, develop, manufacture and sell medicines for preventive or curative treatments. The public health is directly at stake that implies the important regulation and supervision by regulatory authorities, guaranteeing the safety, efficacy, and quality of the potential new medicines. A drug development project is defined as a process of knowledge acquisition that allows a presumably active chemical or biological entity to become a pharmaceutical drug. After passing a series of tests, the drug is certified for commercialization, guaranteeing its safety, efficacy, and quality (Gourc and Bougaret (2000)). The tests help characterizing the behavior of the new compound, its absorption, distribution, metabolism, mechanism of action, side effects, and elimination from the human body, for diverse age groups and patient populations. The availability and mode of production also have to be studied. These tests and studies are numerous and time-consuming. Thus the pharmaceutical $\mathrm{R} \& \mathrm{D}$ projects last over 13 years and cost more than $\$ 1$ billion - higher than NASA's budget for sending a rocket to the moon (Garnier (2008)). The tests and studies are organized in different phases separated by Go/No Go decision milestones, wherein a steering committee decides whether to continue the project if the results are good enough. The success rate is only 4\% (Paul et al. (2010)). The Go / No Go decisions are based on the project status information and results of the tests and studies that are generally not conclusive. Therefore the health public and also the important investments are at stake and it might be difficult to make Go / No Go decisions.

Modeling a generic process The pharmaceutical industry has been relatively slow to implement project management techniques in comparison with other industries (Kennedy (1998)). Interviews show that in the studied pharmaceutical enterprises, this process has not been clearly defined and modeled. Main views of the generation axis of the CIMOSA cube help model decision-making process in drug development projects, presented in figure B.1 in appendix.

Identification of the axes of interviews Go/No Go decisions are based on information about the properties of the potential new medicine. Information has an important role to shape decisions and is one axis of the interview grid. Despite a large number of the tests on the molecule, the degree of uncertainty is high. Uncertainty is another axis of the grid. The non-emergency axis deals with the impact of time perception on decision-making. The characteristics and culture of enterprises have an important impact on the ways of taking decisions and form an axis of the grid. The individual and collaborative factors are also taken into account in the last axis.

Identification of the target actors Several interviews with seven actors of French pharmaceutical industry have been done. The interviewees are junior, senior, and retiree. They are pharmacologists, chemists, doctors, and managers in three main roles: 1) steering committee's members who make decisions about projects and have to be convinced of the interest of projects, 2) project managers who present the project status information and the results of the studies to the steering committee, and 3 ) planners / coordinators who help manage projects. The first group make Go / No Go decisions and the second and third group provide the the first group with information that they need to decide. All seven interviewees played at least one of these roles during their career. They are the pharmacologists, chemists, doctors, and managers who occupied the jobs such as R\&D director, Senior-Director of Chemical and Pharmaceutical Development, Head of Process Investigation / Process Research, Director of Preindustrial and Clinical phase, Head of Portfolio Management Consultant (Experts), Project Manager, and Project Coordinator in pharmaceutical companies. Most of the interviewees have been working more than one company during their career: 3 French pharmaceutical companies, 1 Dutch company, 1 European company, 1 American company with 2000-10,000 employees.

Development of the interview grid Here are the main topics of the questions of each axis: 1) information: 11 questions about the importance of presentation of information by project manager to decision-makers, its quality, quantity, objectivity, and accessibility for the decisionmakers of different fields, 2) uncertainty: 5 questions about its frequency, sources, impacts on decisions and the ways to handle it. 3) none-emergency of situations: a question on the impact of non-emergency on decision-making. 4) culture of the enterprise: 5 questions about the type of governance, structure and functioning of decisional system, flow of information, and other implicit/explicit rules. 5 ) individual and collaborative factors: 4 questions about the influences of the group on individuals and vice versa.

Validation of the grid The main axes of our grid have been validated by one of the actor who helped to reformulate some questions in order to be clearer, using the key words of the filed such as benefit-risk balance, decision recycling, jargon translator. In the axis of information 
some questions on the quality and time of the presentation of information are added.

Interviews The interviews last an average of four hours and some interviewees are solicited by email to complete their opinions and some of them contacted us to add other reflections or the points of view of their colleagues.

Identification and structuring the efficient practices The efficient practices are helpful to a successful implementation of the process. They are structured in three main classes: subject, object, and context. Some brief examples are as follows.

The subject class regroups the practices related to the individual and collaborative properties of the industrial actors. At individual level, it is important to accept uncertainty. Decision-making in R\&D faces much uncertainty in all industries, since "innovation is one of the causes of uncertainty", an interviewee says. In pharmaceutical industry, the degree of uncertainty is particularly high, since the behavior of the new compounds in the human body is not completely verifiable. At collaborative level, it is important to avoid conformity in meetings, to express in order to avoid the false imagination of other people's opinion, and to avoid misunderstandings caused by silence. These practices are studied by Morel (2002) in the final decision to launch Challenger shuttle. In drug development projects, "some individuals do not express their disagreements during the meeting and try to influence the decision after the meeting", says an interviewee. This behavior harms the transparency in an enterprise. The relation between an individual and a group should be reciprocal. An individual has to listen, but also express and argue during a meeting. These practices may reduce the invalidation of decisions a posteriori. Some organizational practices may favor these collaborative practices. Some examples are given in the contextual factors.

The object class regroups the practices related to management of the information and uncertainties about the project. One of the efficient practice in presentation of the results of studies by project managers is to adapt the contents to the knowledge level of interlocutors (decisionmakers). The composition of the steering committee has to be taken into account by the project manager. The level of the interpretation of raw data must depend on the composition of the steering committee. If the members of the steering committee are mostly scientific, the project manager can give for example, a number as a degree of the stability or toxicity of a molecule. If the composition of the steering committee is mostly marketing and financial, the project manager must give the clear interpretation and contextualization of the raw data, not only a number: for example, the molecule is toxic but compared to its class and the target indication (cancer), this degree of toxicity is acceptable. Another efficient practice related to the information is to limit the details, since the results of the tests and studies are very voluminous. For example, the electrocardiogram of the nth rat has not be presented by the project manager neither asked by the steering committee in a Go / No Go decision meeting.

The context class regroups the practices related to organisation, governance and culture of enterprise. "In a company, we know or do not know how to make decisions and it is the culture of the enterprise that determines it", says an a project manager. All the interviewees agree about the importance of the culture and governance of the enterprise on the behavior of the people who make or help decisions. The global strategy of an enterprise must be clear in order to reduce delays in decision-making, since decisions are based on: the information about the scientific and marketing factors of the projects, and the goals of the decision-makers. These goals should be determined by the strategy of the enterprise. "We must know what we would obtain and what we would pay for it. We must know how to make a balance between scientific and marketing factors", affirm an interviewee. Another proposes to link these strategies directly to the innovation pole: "in order to favor innovation, it should not exist an intermediate hierarchical level between the general direction and the innovation pole." In organization of decisions, it is important to avoid tele-decision-making. "Today, Internet allows to communicate easily via video-conferencing. Teledecision-making is not a good practice in decision-making, since in pharmaceutical industry, even if all the members of the steering committee are around the same table to discuss and decide, it is usually difficult to understand each other and make a decision, since the projects are complex", affirm an interviewee. "All decision is a matter of compromise" (Simon (1947)). A collaborative decision can be made reaching a compromise either between all decision-makers, or the majority of them, or some of them who have more experience, influence or power. "To make decisions in due time, it should be clear who really make/s the final decisions", affirm an interviewee. It is important to specify the goals of each meeting at the beginning and to conclude by summarizing the decisions at the end. "It can happen that at the end of a meeting, after the controversy debates, we do not really know whether a decision is made or not! We have to specify what the decision is about at first and announce the issue of the decision at the end", affirm an interviewee. After making a decision, it is important to communicate the decision to all the members of the project team. It helps the execution of the decisions. "It can happen that a decision is made by the steering committee, but it is not communicated and explained to the people who have worked for the project. It is not respectful for these people", affirm an interviewee. Make and communicate a decision is not enough. After that, decisions should be respected. "If all members of the steering committee participate in decision-making and good interactions exist within a group, the decision will be respected and executed. If not, it is possible to invalidate it frequently and we never know when it is really made!", says an interviewee. Both positive and negative feedbacks from previous decisions can be useful for the future decisions. It is important to store a summary of the way decisions are made. These practices are principally destined to project managers, decision-makers (members of steering committee), and the general direction, in three times: before, during, and after decision meeting and could help making decisions more quickly and effectively.

These practices are the feedback of many years of experience of the actors and correspond to a successful period of an enterprise's life. This first version of the practices contain only the practices that are recommended by all 
interviewees. The proposed practices are reviewed and validated by the experts.

\section{CONCLUSIONS AND PROSPECTS}

Delays in decision-making adversely affects reaching the goals of the industrial projects on quality, time, cost, and respecting human well-being. The purpose of this paper is to propose a methodology to identify influential factors and efficient collaborative practices in decision-making process in industrial projects. The proposed methodology helps characterize the indecision problem in a given industrial context, provide a generic process as an enterprise reference model, an interview grid, and a typology of efficient practices.

A case study in pharmaceutical industry is presented. Through the proposed approach, decision-making in drug development projects is modeled. A grid of interview is developed. Seven interviews are performed to identify a set of efficient practices that are the feedback of many years of experience of the actors. The practices may help to reduce the delays in making-decision.

The proposition of efficient practices is not enough to improve the efficiency of an enterprise. The actors have to be prepared and conducted to changes in their practices. Therefore, a prospect of this work is to identify the barriers to efficient practices, to develop an action plan of changes and to adopt a change management in order to assist the actors in changes.

\section{ACKNOWLEDGEMENTS}

The authors express thanks to all interviewees for sharing their experience and opinions.

\section{REFERENCES}

AMICE (1993). CIMOSA: open systems architecture for CIM. Springer-Verlag.

Bacanli, F. (2006). Personality characteristics as predictors of personal indecisiveness. Journal of Career Development, 32(4), 320-332.

Darras, F. (2004). Proposition d'un cadre de référence pour la conception et l'exploitation d'un progiciel de gestion intégré. Ph.D. thesis, Institut National Polytechnique de Toulouse.

Davenport, T.H. (2009). Make better decisions. Harvard Business Review.

Davenport, T.H. (2010). How organizations make better decisions. International Institute for Analytics.

Denis, J., Dompierre, G., Langley, A., and Rouleau, L. (2011). Escalating indecision: between reification and strategic ambiguity. Organization Science, 22(1), $225-244$.

Ferchichi, A. (2008). Contribution à l'intégration des processus métier : application à la mise en place d'un référentiel qualité multi-vues. Ph.D. thesis, Ecole Centrale de Lille Ecole Centrale Paris.

Garnier, J. (2008). Rebuilding the R\&D engine in big pharma. Harvard Business Review.

Germeijs, V. and De Boeck, P. (2002). A measurement scale for indecisiveness and its relation to career indecision and other types of indecision. European Journal of Psychological Assessment, 18(2), 113-122.
Gourc, D. and Bougaret, S. (2000). L'industrie pharmaceutique : ses projets de développement, leurs caractéristiques et leur management. La cible, la revue du management de projet, 15ème année(81), 4-8.

Hammer, M. and Champy, J. (1993). Le reengineering. Dunod.

Hassanzadeh, S., Gourc, D., Marmier, F., and Bougaret, S. (2012). Decision-making under uncertainty in drug development. Project Perspectives, The annual publication of IPMA.

Kennedy, T. (1998). Pharmaceutical project management. Marcel Dekker.

Morel, C. (2002). Les Décisions absurdes. Gallimard.

Paul, S.M., Mytelka, D.S., Dunwiddie, C.T., Persinger, C.C., Munos, B.H., Lindborg, S.R., and Schacht, A.L. (2010). How to improve R\&D productivity: the pharmaceutical industry's grand challenge. Nature Reviews Drug Discovery, 9(3), 203-214.

Simon, H.A. (1947). Administrative behavior: a study of decision-making processes in administrative organizations. Free Press.

Vernadat, F. (1996). Enterprise modeling and integration: principles and applications. Springer.

\section{Appendix A. MAIN STEPS OF THE PROPOSED METHODOLOGY}

\section{Appendix B. GENERIC DECISION-MAKING} PROCESS 


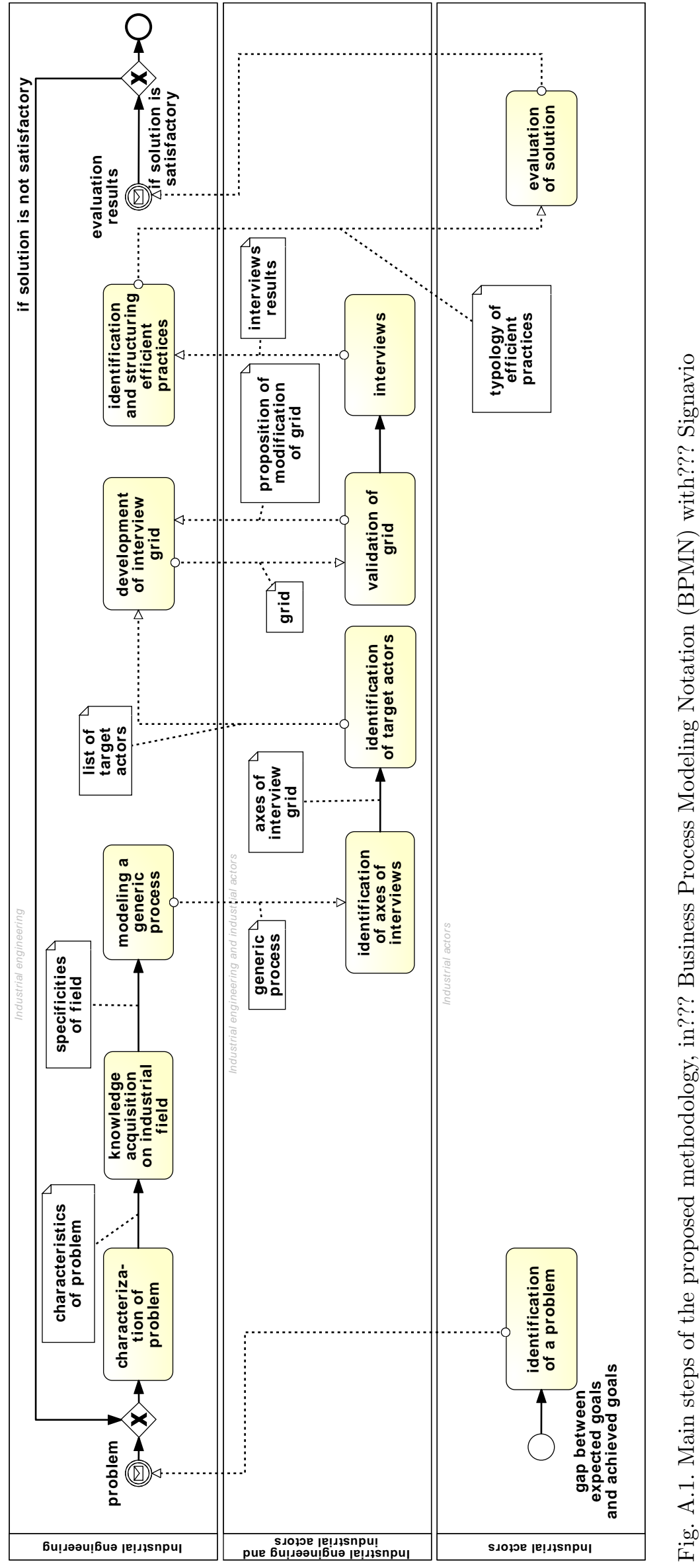




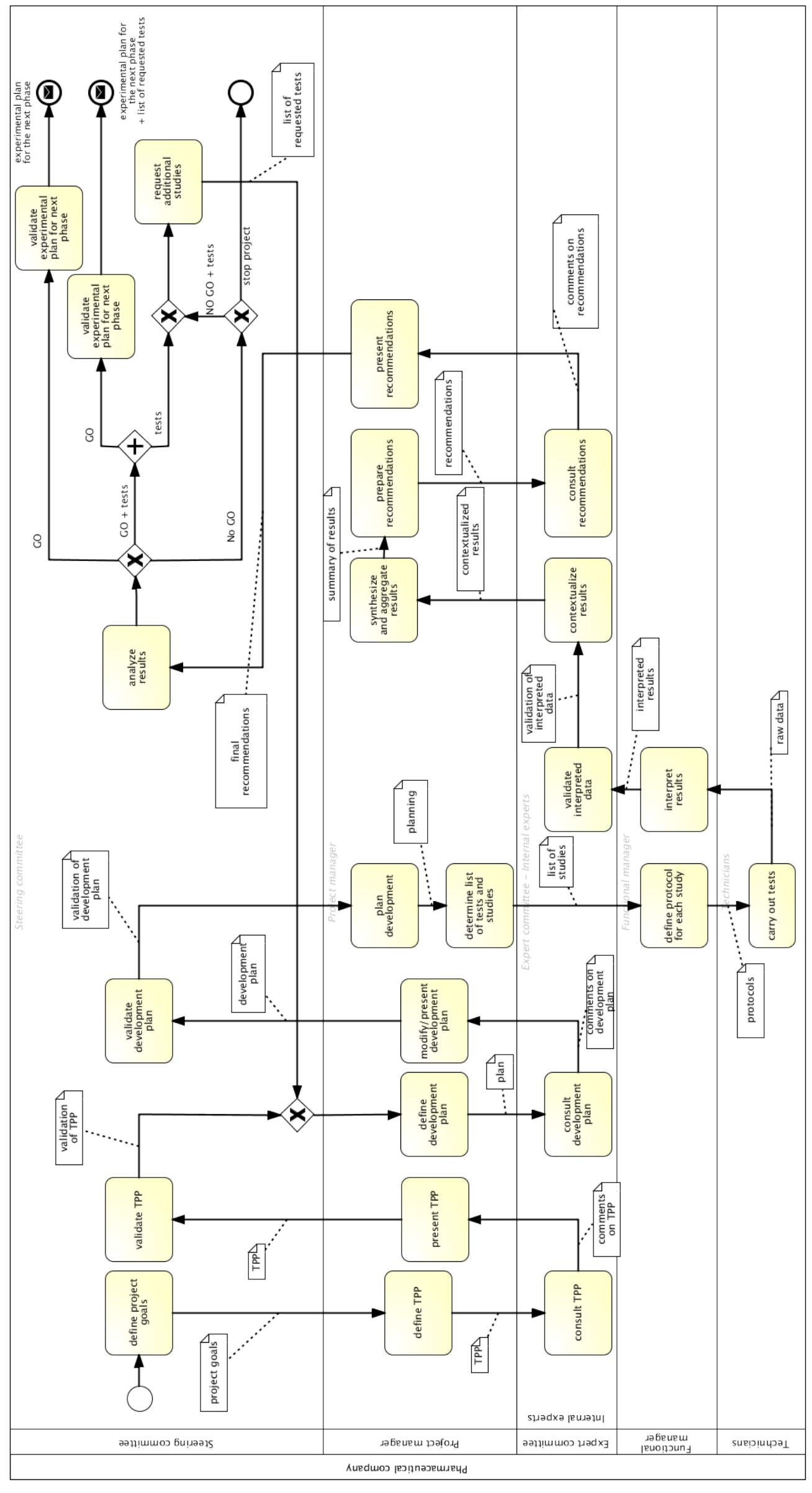

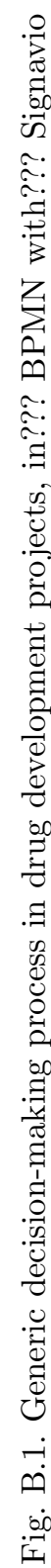

